\title{
Superior ophthalmic vein and ophthalmic artery in immediate evaluation after endovascular treatment of carotid-cavernous fistulas
}

\author{
Krzysztof Brzozowski ${ }^{A, B, D, E}$, Jerzy Narloch ${ }^{B, C, D, E, F}$, Piotr Zięcina ${ }^{D, F}$, Andrzej Podgórski ${ }^{D, E}$, Piotr Piasecki ${ }^{B, D, E, F}$ \\ Military Institute of Medicine, Warsaw, Poland
}

\section{Abstract}

Purpose: To validate superior ophthalmic vein (SOV) and ophthalmic artery (OA) usefulness in immediate evaluation of new endovascular approaches to treat carotid-cavernous fistulas (CCFs).

Material and methods: A retrospective review of 597 intracerebral malformation embolisations yielded 40 embolisations of CCF in the treatment of 18 patients. Two interventional radiologists performed detailed radiological angiographic assessments.

Results: Mean age at initial admission was 58.9 years (SD 18.5 years, range $24-85$ years). Patients presented with: chemosis (50\%), ocular bruit (50\%), exophthalmos (61\%), diminished visual acuity $(77.8 \%)$, headache (16.7\%), and intracerebral haemorrhage (5.55\%), and 5.55\% were asymptomatic. Unilateral fistulas (10-55.5\%) showed more diversified venous drainage pattern than bilateral ones (8-44.4\%). There were statistically significant differences in post-traumatic and spontaneous CCF regarding age $(p=0.036)$, type of fistula $(p=0.0008)$, and presence of pseudoaneurysm $(\mathrm{p}=0.036) .77 .8 \%$ of patients had increased ipsilateral SOV diameter. SOV enlargement was not associated with type of fistula, history of trauma, or degree of exophthalmos. Ipsilateral ophthalmic artery was visible in all patients on both pre- and postprocedural angiography on lateral projection. Pre- and post-procedural SOV diameter was significantly different. Internal carotid artery patency was $100 \%$, while the overall final angiographic or clinical success was $85.7 \%$. We had three cases of peri-procedural complications.

Conclusions: We reported changeable dynamics of SOV and OA after endovascular treatment of CCFs and proved the feasibility of coils and Onyx-18 in the treatment thereof.

Key words: CCF, interventional neuroradiology, embolisation, coils, Onyx.

\section{Introduction}

Carotid-cavernous fistula (CCF) is an abnormal communication between the internal carotid artery (ICA), external carotid artery (ECA), or any of their branches with the cavernous sinus. These lesions are usually classified as direct or indirect. Direct fistulas have an abnormal communication between the ICA and the cavernous sinus. Indirect fistulas have an abnormal communication between the meningeal branches of the ICA or ECA and the cavernous sinus [1].
The classic triad of exophthalmos, ocular bruit, and episcleral congestion is secondary to reversal of blood flow in the ophthalmic veins. Although the principal manifestations of venous hypertension are ophthalmic, cranial nerves entering the superior orbital fissure could also be affected, leading to ocular symptoms. Among possible presentations are bleeding from the mouth, nose, or ears, intracranial haemorrhage, increased intracranial pressure, and steal phenomena [2].

The treatment of CCF depends on the severity of the clinical symptoms, its angiographic properties, and the

Correspondence address:

Jerzy Narloch, Military Institute of Medicine, 128 Szaserow St., 04-141 Warsaw, Poland, e-mail: jerzy.narloch@gmail.com

Authors' contribution:

A Study design · B Data collection · C Statistical analysis · D Data interpretation - E Manuscript preparation · F Literature search · G Funds collection 
risk it presents for intracranial haemorrhage. Treatment is recommended in patients with symptoms refractory to medication or with cortical or deep venous reflux. The first-line approach is endovascular embolisation with detachable balloons, coils, glue, covered stents, or a combination of the above [2].

Advances in endovascular technology have given rise to novel treatment options, successively utilised in different pathologies. Long-established techniques are gradually exchanged or complemented with new hardware, creating new standards of treatment. Immediate assessment of treatment efficacy is crucial. Initial evaluation post-treatment is problematic because the clinical symptoms subside gradually, even if the fistula is embolised completely. In this paper we aimed to assess the superior ophthalmic vein (SOV) and ipsilateral ophthalmic artery (OA) characteristics for treatment evaluation after new endovascular approaches.

\section{Material and methods}

The study was conducted in a department of interventional radiology of tertiary care referral centre. We retrospectively reviewed our patient database from January 2008 and December 2016. During this time, we performed 597 intracerebral malformation embolisations, 40 of which were CCF embolisations in the treatment of 22 fistulas in 18 patients.

Two experienced interventional neuroradiologists evaluated the radiological data of all patients. Data included: SOV diameter measurement - measured in its wider diameter on lateral projection on both sides. It was considered enlarged if it exceeded $3 \mathrm{~mm}$; exophthalmos was recorded as a distance between the interzygomatic line (a line drawn at the anterior portions of the zygomatic arches) to the anterior surface of the globe on axial images (nonenhanced computed tomography - CT or magnetic resonance imaging - MRI); time, absorbed dose, and contrast volume used for each procedure; type of embolic agent; other intracranial vascular malformation; visibility of ipsilateral optic artery pre- and post-procedure; venous drainage pattern. Eventually the fistulas were classified according to Barrow [1].

All patients were operated under general anaesthesia. Intra-operative monitoring included heart rate, electrocardiogram, oxygen saturation, end tidal carbon dioxide, urine output, and arterial blood gas analysis. Any sudden change in the heart rate of at least $20 \%$ of the baseline was considered significant.

Careful management of coagulation was done to prevent thromboembolic complications during and after the procedure. After arterial access was gained, intravenous heparin (50 IU kg-1) was given in a bolus with an additional bolus of $1000 \mathrm{IU}$ at least every hour. A heparin infusion was continued throughout the procedure through the side channel of the femoral sheath to protect against thrombogenic effects of endothelial trauma and the inherently thrombogenic nature of the materials used, which can cause retrograde thrombosis in embolised vessels.

Transfemoral access using a 6-7 Fr femoral sheath was achieved. Then, an angiographic catheter was introduced along with a soft-tip guide wire, and diagnostic bilateral selective ICA, ECA, and vertebral artery angiography was performed in all patients. Regarding angiographic characteristics, we analysed the size and location of the fistula, the presence of any associated ICA aneurysm, pseudoaneurysm, cortical venous drainage, cavernous sinus ectasia, the feeding arteries and the pattern of venous drainage, and the morphology of the common carotid arteries (CCA) origin and bifurcation, after which appropriate hardware was selected (coils - Axium [ev3, Irvine, CA] or liquid embolic agent - Onyx-18 [Micro Therapeutics Inc., Irvine, CA]).

Next, a 5 Fr guiding sheath was placed in the ICA in a stable position. A hardware compatible microcatheter was advanced into the fistulous point/s under roadmap guidance. The fistula was considered to be embolised satisfactorily once there was angiographic obliteration of the fistula or when there was reversal of steal and reappearance of the ipsilateral ophthalmic artery on angiography.

Statistical analysis was performed using Statistica (StatSoft, Inc., Tulsa, OK, USA). Descriptive statistics of all variables were calculated. Normally distributed quantitative variables were compared using Student's $t$-test between patients, depending on the material used and whether the patient sustained trauma or not; non-normally distributed categorical variables were compared using the $U$-Mann Whitney test. Wilcoxon paired test was used to compare SOV diameter pre- and post-treatment (Figure 1). Correlations between variables were calculated using Spearman's rank correlation coefficient. The statistical level of significance was set at $p=0.05$.

\section{Results}

Eighteen patients ( 10 women and 8 men) with a mean age at initial admission of 58.9 years (SD 18.5 years, range 24-85 years) presented the following: nine (50\%) showed chemosis, nine (50\%) ocular bruit, 11 (61\%) exophthalmos, and $14(77.8 \%)$ complained of diminished visual acuity, which could be partly attributable to third and sixth nerve palsy in three (16.7\%) and six (33.3\%) of them, respectively. Three (16.7\%) patients had a headache, one (5.55\%) had intracerebral haemorrhage, and one (5.55\%) was asymptomatic.

Unilateral fistulas showed more diversified venous drainage pattern than bilateral ones - results are shown in Table 1. There were four patients with bilateral fistulas. Spearman's rank correlation coefficients for the recorded characteristics were presented in Table 2.

The group comprised of nine (41\%) type A, five (22.7\%) type B, and eight (36.3\%) type D fistulas. Type A 

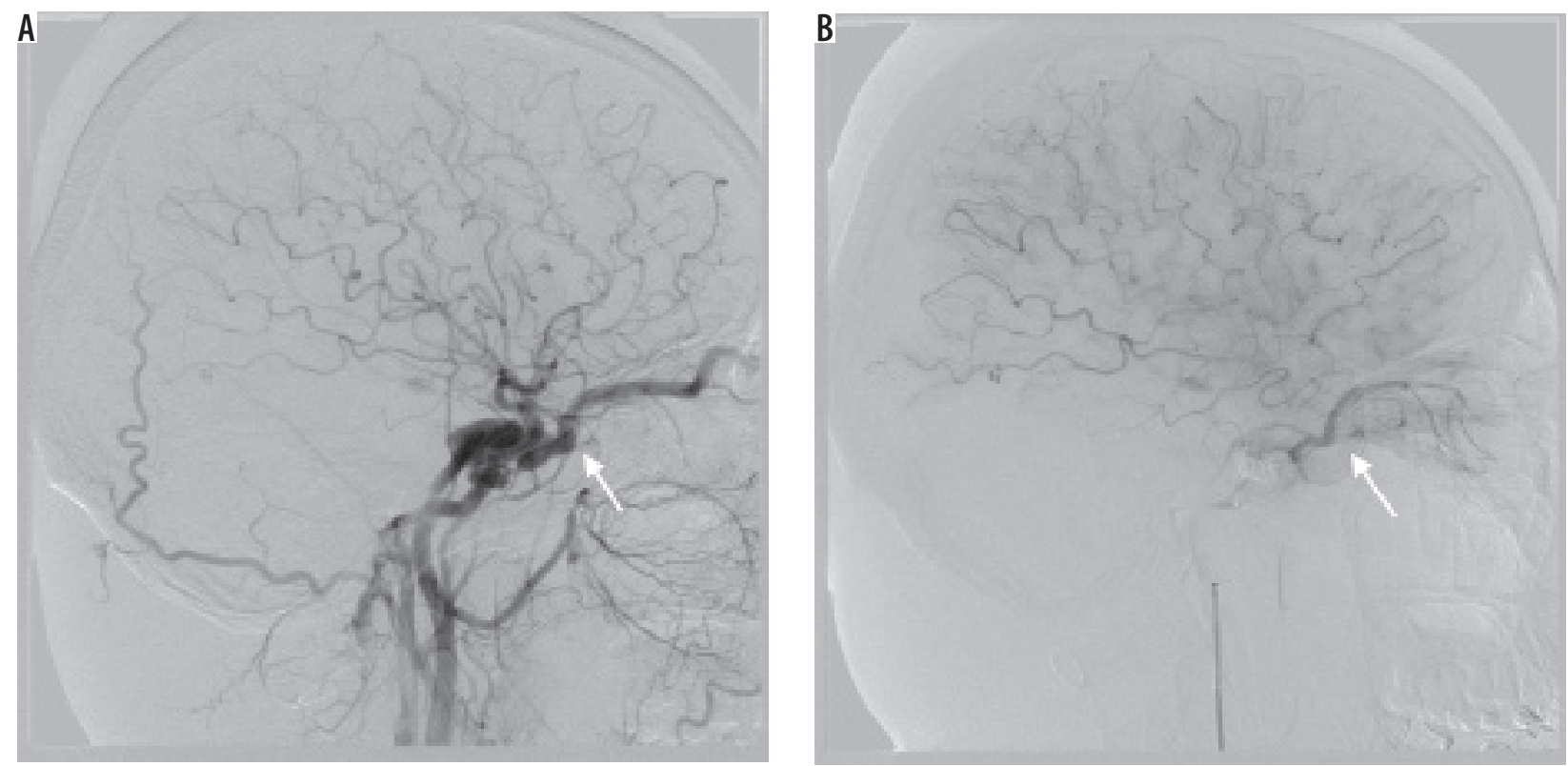

Figure 1. Note the differences in superior ophthalmic vein diameter pre- and post-treatment

was mostly associated with younger age and more contrast material used, although the results did not reach statistical significance. Type A fistulas were more likely to result from trauma $(p=0.004)$, which in consequence were accompanied by post-traumatic pseudoaneurysm at the site of the fistula or elsewhere. Type D fistulas needed more attempts to treat, whereas type B fistulas where least likely to be successfully treated, compared to other types. Table 3 summarises the results of matching different types of fistulas using pairwise Kruskal-Wallis test for multiple comparisons.

There were statistically significant differences in posttraumatic and spontaneous CCF regarding age (in favour of younger patients in trauma-related lesions, $p=0.036$ ), type of fistulas $(77.7 \%$ of type A in traumatic fistulas, $p=0.0008)$, and the presence of pseudoaneurysm $(p=0.036)$. Trauma-related lesions consumed more contrast medium in the treatment process $(p=0.002)$ and were clinically more conspicuous, i.e. by the degree of exophthalmos $(p<0.05)$. There were no differences with regard to other procedure-related resources, such as mean time or dose absorbed during the procedure ( $p=0.413$ and $p=0.108$, respectively) or number of attempts needed to treat the fistula $(p=0.954)$. The analysis showed no statistically significant differences of the recorded characteristics when the type of embolisation material used divided the group.

Fourteen (77.8\%) patients had increased ipsilateral SOV diameter (mean $4.05 \mathrm{~mm}, \mathrm{SD} 1.16 \mathrm{~mm}$, range 3.24-6.6 mm). The contralateral SOV diameter was on average $1.46 \mathrm{~mm}$. The ipsilateral SOV diameter immediately after treatment was $2.08 \mathrm{~mm} \pm 1.6 \mathrm{~mm}$. SOV enlargement was not associated with type of fistula, history of trauma, or degree of exophthalmos (Table 2). The SOV diameter was significantly associated ipsilaterally with cavernous sinus venous drainage $(p<0.0001)$, which in most cases involved reflux to SOV itself (54\% of unilateral and $100 \%$ of bilateral
Table 1. Venous drainage pattern - comparison between unilateral and bilateral carotid-cavernous fistulas

\begin{tabular}{|l|c|c|}
\hline & Unilateral & Bilateral \\
\hline Ipsilateral cavernous sinus & 7 & - \\
\hline Bilateral cavernous sinus & 4 & 8 \\
\hline Superior ophthalmic vein & 6 & 8 \\
\hline Inferior ophthalmic vein & 1 & 0 \\
\hline Straight sinus & 2 & 0 \\
\hline Sigmoid sinus & 1 & 0 \\
\hline Ipsilateral cortical veins & 1 & 0 \\
\hline
\end{tabular}

fistulas). The remaining venous drainage patterns were not associated with SOV diameter. SOV was ipsilateral ophthalmic artery was visible in all patients on both pre- and post-procedural angiography on lateral projection. Preand post-treatment ipsilateral SOV measurements were significantly different in Wilcoxon paired test $(p=0.011)$. The SOV diameter, regardless of the stage of treatment, was not significantly correlated with the result of the embolisation. In all cases there was normalisation of flow direction after the procedure.

ICA patency was $100 \%$, while the overall final angiographic or clinical success was $85.7 \%$ after a median of two attempts (SD 1.2, range 1-4). In three patients, we failed to achieve arterial access due to small diameter of the feeder(s), in one there was no clear fistulous point and the fistula was extremely small, and in one patient the fistula had recanalised. In treatment of CCFs we used either coils $(72 \%)$ or Onyx-18 (28\%), with success rates of $92.3 \%$ vs. $62.5 \%$, respectively (we assumed those that we failed to microcatheterise were bound to be treated with Onyx-18, and were marked as failures in our calculations). The successful outcome of the treatment was significantly asso- 
Table 2. Spearman's rank correlation coefficients. Marked values were statistically significant $(p<0.05)$. Class. refers to Barrow classification category; SOV was recorded as a diameter in $\mathrm{mm}$; Time, Dose, and Contrast were mean values per fistula and were recorded as min, $\mathrm{mGy}$, and ml, respectively; Result refers to closure of the fistula; Material refers to either coils or Onyx-18; Exophtalmos was recorded as a distance between the interzygomatic line (a line is drawn at the anterior portions of the zygomatic arches) to the anterior surface of the globe on axial images

\begin{tabular}{|c|c|c|c|c|c|c|c|c|c|c|c|c|c|c|}
\hline & gั & ๗ั̊ & $\begin{array}{l}\dot{\ddot{c}} \\
\text { d. }\end{array}$ & 흐 & 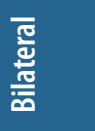 & 兽 & ஜั̆ & 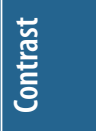 & 늘 & 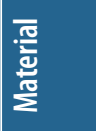 & 吕 & 毫 & 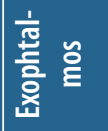 & 흥 츨 \\
\hline Age & & 0.72 & 0.48 & -0.06 & 0.03 & 0.13 & 0.01 & -0.30 & -0.71 & 0.42 & -0.57 & -0.40 & 0.06 & -0.24 \\
\hline Sex & 0.72 & & 0.27 & -0.07 & 0.24 & 0.14 & -0.08 & -0.12 & -0.43 & 0.100 & -0.58 & -0.03 & -0.72 & 0.09 \\
\hline Class. & 0.48 & 0.27 & & 0.28 & 0.46 & -0.24 & -0.42 & -0.63 & -0.17 & 0.49 & -0.85 & 0.07 & 0.12 & -0.67 \\
\hline SOV & -0.06 & -0.07 & 0.28 & & 0.25 & -0.30 & -0.33 & -0.47 & -0.06 & 0.06 & -0.26 & -0.003 & 0.13 & -0.19 \\
\hline Bilateral & 0.03 & 0.24 & 0.46 & 0.25 & & 0.09 & -0.04 & -0.15 & 0.04 & -0.07 & -0.33 & 0.41 & -0.24 & -0.04 \\
\hline Time & 0.13 & 0.14 & -0.24 & -0.30 & 0.09 & & 0.93 & 0.58 & -0.08 & 0.09 & 0.25 & 0.02 & -0.36 & 0.36 \\
\hline Dose & 0.01 & -0.08 & -0.42 & -0.33 & -0.04 & 0.93 & & 0.55 & 0.00 & 0.12 & 0.47 & 0.06 & 0.08 & 0.39 \\
\hline Contrast & -0.30 & -0.12 & -0.63 & -0.47 & -0.15 & 0.58 & 0.55 & & -0.05 & -0.13 & 0.88 & -0.23 & 0.06 & 0.69 \\
\hline Result & -0.71 & -0.43 & -0.17 & -0.06 & 0.04 & -0.08 & 0.00 & -0.05 & & -0.13 & 0.56 & 0.49 & -0.29 & 0.24 \\
\hline Material & 0.42 & 0.10 & 0.49 & 0.06 & -0.07 & 0.09 & 0.12 & -0.13 & -0.13 & & -0.22 & -0.31 & 0.44 & -0.23 \\
\hline Trauma & -0.57 & -0.58 & -0.85 & -0.26 & -0.33 & 0.25 & 0.47 & 0.88 & 0.56 & -0.22 & & -0.03 & -0.13 & 0.74 \\
\hline Attempts & -0.40 & -0.03 & 0.07 & -0.003 & 0.41 & 0.02 & 0.06 & -0.23 & 0.49 & -0.31 & -0.03 & & -0.61 & 0.18 \\
\hline Exophtalmos & 0.06 & -0.72 & 0.12 & 0.18 & -0.24 & -0.36 & 0.08 & 0.06 & -0.29 & 0.44 & -0.13 & -0.61 & & -0.33 \\
\hline $\begin{array}{l}\text { Pseudo- } \\
\text { aneurysm }\end{array}$ & -0.24 & 0.09 & -0.67 & -0.19 & -0.04 & 0.36 & 0.39 & 0.69 & 0.24 & -0.23 & 0.74 & 0.18 & -0.33 & \\
\hline
\end{tabular}

ciated with post-traumatic lesions, in patients of younger age, and after multiple attempts (Table 2).

We had three cases of peri-procedural complications - one patient developed a thromboembolic event, successfully treated with intraarterial thrombolysis with no clinical consequences in the follow-up, another had an aggravation of ocular symptoms immediately after first session and eventually needed urgent interventions to treat the fistula (uneventful on follow-up - $m R S=0$ ), and one had a persistent mild abducens palsy $(\mathrm{mRS}=1)$ that did not resolve until three-month follow-up. One of the patients died due to other sustained injuries.

\section{Discussion}

Classic presentation of CCFs is attributed to venous congestion and concomitant neurological deficits of cranial nerves responsible for eye movement. Successful treatment could be evaluated either radiologically or clinically. From the radiologist's perspective, immediate evaluation based on control angiography would be the most desirable method. We tried to evaluate whether SOV or ophthalmic artery (for the cases of high-flow fistulas) could successfully serve this purpose.

In our series four (22\%) patients had no SOV enlargement with classic orbital symptoms, which is substantially higher than reported before [3]. Alternative venous outflow pathways, providing partial decompression of SOV, or no radiological distention of SOV regardless of high blood flow, could explain signs of orbital venous congestion without SOV distention. We can confirm that the first explanation was the case in our group. Moreover, we found that in one of these patients there was substantial oedema of orbital tissues (seen on angiography, especially regarding orbital muscles), leading to their enlargement, which could potentially prevent enlargement of SOV despite elevated orbital venous pressure (Figure 2).

There is some controversy regarding the cut-off point for the SOV diameter to be considered enlarged, because it was reported to be highly variable, and asymmetric within the individuals. Mean normal diameters are ranging from 0.3 to $4.6 \mathrm{~mm}[4,5]$. For the purpose of this study we adopted the value of $3 \mathrm{~mm}$ as a threshold, after Ozgen $e t$ al. [4]. The vast majority of SOV measurements reported in literature come from axial MR images, while we performed the measurements on lateral projection on cerebral angiography, which might also lead to inconsistencies. One of the reasons was that MR or CT axial images were not available for all patients, mostly because we wanted to utilise SOV distention decrease as a sign of successful embolisation. Unfortunately, immediately after fistula closure (obliteration on control angiography) we frequently observed normalisation of flow direction, and velocity with no actual decrease in SOV distention. In two cases, we actually observed a marked increase in the diameter (1.94 vs. $3.28 \mathrm{~mm}$ and $1.1 \mathrm{~mm}$ vs. $2.43 \mathrm{~mm}$ ). In the first case, there was normalisation of flow velocity and direction, from high- to low-flow, and resolution of 
venous reflux. The patient had no clinical adverse event post-treatment. The second patient died of sustained injuries - a detailed description is provided under Figure 3.

Ipsilateral ophthalmic artery was visible in all patients on both pre- and post-procedural angiography on lateral projection regardless of type of fistula, which is contrary to the recent report by Joshi et al. [6]. In a collection of posttraumatic CCFs they did not observe ipsilateral ophthalmic artery in $76 \%$ of patients.

Treatment is indicated in symptomatic patients, with lesions leading to visual impairment - usually due to ocular or orbital involvement, or cranial nerve deficits - cortical venous drainage, or haemorrhage [2]. CCFs can present themselves as clinically stable lesions or with precipitating symptomatology, which is reported to be associated with spontaneous venous thrombosis in part of the outflow [7-9]. Apparently, further investigation is necessary to evaluate the usefulness of possible ophthalmic artery reappearance after successful embolisation.

Worsening of symptoms could also occur after initial endovascular therapy, and could be directly related to a change of venous outflow pattern, with redirection of blood flow prompting congestion of the remaining part [10]. One of our patients (27-year-old male, with no history of head trauma) with a high-flow type A fistula, who was initially admitted due to severe ocular symptoms, underwent first embolisation of the fistula using coils, with initial closure of the fistula on angiography. After two days ocular symptoms aggravated, which led to prompt cerebral angiography and the realisation that the fistula had recanalised partially, with venous outflow directed solely to superior ophthalmic vein. The remaining fistula was successfully embolised with coils, which eventually led to resolution of all symptoms on follow-up after three months post-embolisation. On pre-treatment evaluation, there was mild ipsilateral abducens palsy, which worsened after initial treatment but subsided three months after final embolisation. The prolonged clinical deficit could in this case be directly related to compression of the nerve by coils implanted in the cavernous sinus.

Six of our patients had abducens nerve deficit of varied intensity, often coexisting with oculomotor palsy. In two of them the symptoms persisted, but the mild deficit was clinically conspicuous on three-month follow-up only in one case. These complications are unremarkable compared to other studies in which cranial nerve deficits were the most common complications reported [11,12]. The coils may, due to progressive thrombosis of the cavernous sinus, mass effect, or direct injury of the nerve, cause the complications [13]. The anatomical location of the abducens nerve could explain its most frequent involvement $[11,13]$.

Our overall success rate via transarterial access was $85.7 \%$, which is comparable to other reports; however, we managed to maintain ICA patency in all patients (59-88\% in the literature) $[6,9,11-14]$. The main reason could be the
Table 3. Results of the Kruskal-Wallis test - differences ( $p$ values) between types of fistulas. Significance level $p<0.05$

\begin{tabular}{|c|c|c|c|}
\hline \multicolumn{4}{|c|}{ Age } \\
\hline & A & B & D \\
\hline$A$ & & 0.117765 & 0.094085 \\
\hline$B$ & 0.117765 & & 1.000000 \\
\hline$D$ & 0.094085 & 1.000000 & \\
\hline
\end{tabular}

\section{SOV}

\begin{tabular}{|l|c|c|c|} 
& A & B & D \\
\hline A & & 1.000000 & 1.000000 \\
\hline B & 1.000000 & & 1.000000 \\
\hline D & 1.000000 & 1.000000 & \\
\hline
\end{tabular}

\section{Time}

\begin{tabular}{|l|c|c|c|}
\hline & A & B & D \\
\hline A & & 0.538661 & 1.000000 \\
\hline B & 0.538661 & & 1.000000 \\
\hline D & 1.000000 & 1.000000 & \\
\hline
\end{tabular}

\section{Dose}

\begin{tabular}{|l|c|c|c|}
\hline & A & B & D \\
\hline A & & 0.172353 & 0.239223 \\
\hline B & 0.172353 & & 1.000000 \\
\hline D & 0.239223 & 1.000000 & \\
\hline
\end{tabular}

\begin{tabular}{|l|c|c|c|}
\hline Contrast & A & B & D \\
\hline A & & 0.172354 & 0.055806 \\
\hline B & 0.172354 & & 1.000000 \\
\hline D & 0.055806 & 1.000000 & \\
\hline
\end{tabular}

\begin{tabular}{|l|c|c|c|}
\hline Attempts & A & B & D \\
\hline A & & 0.152042 & 1.000000 \\
\hline B & 0.152042 & & 0.083094 \\
\hline D & 1.000000 & 0.083094 & \\
\hline \multicolumn{5}{|l|}{} \\
\hline \multicolumn{1}{|l|}{ Result } & A & B & D \\
\hline A & & 0.109267 & 1.000000 \\
\hline B & 0.109267 & & 0.052341 \\
\hline D & 1.000000 & 0.052341 & \\
\hline
\end{tabular}

\section{Pseudoaneurysm}

\begin{tabular}{|l|c|c|c|}
\hline & A & B & D \\
\hline A & & 0.128697 & 0.060357 \\
\hline B & 0.128697 & & 1.000000 \\
\hline D & 0.060357 & 1.000000 & \\
\hline
\end{tabular}

\section{Trauma}

\begin{tabular}{|l|c|c|c|} 
& A & B & D \\
\hline A & & 0.182378 & 0.016840 \\
\hline B & 0.182378 & & 1.000000 \\
\hline D & 0.016840 & 1.000000 & \\
\hline
\end{tabular}

SOV - superior ophthalmic vein diameter

hardware that was used. Most ICA occlusions occurred after endovascular treatment with detachable balloons, which are nowadays almost unavailable. The procedure itself posed a risk of ICA sacrifice due to balloon migration or premature detachment. 

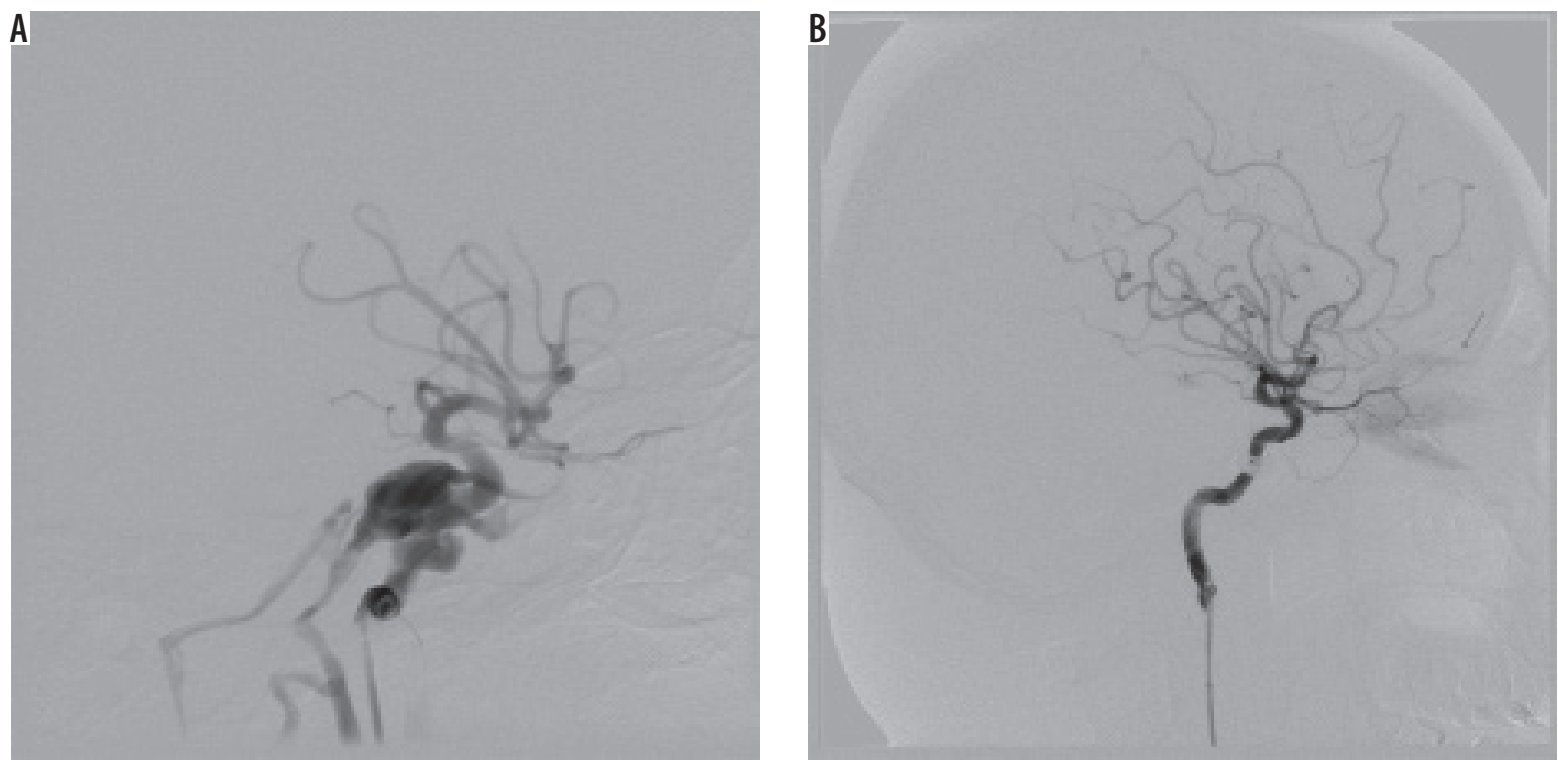

Figure 2. Lateral projection on cerebral angiography - successful embolisation of the post-traumatic type A fistula. Note post-embolisation oedema of orbital muscles (red arrow)
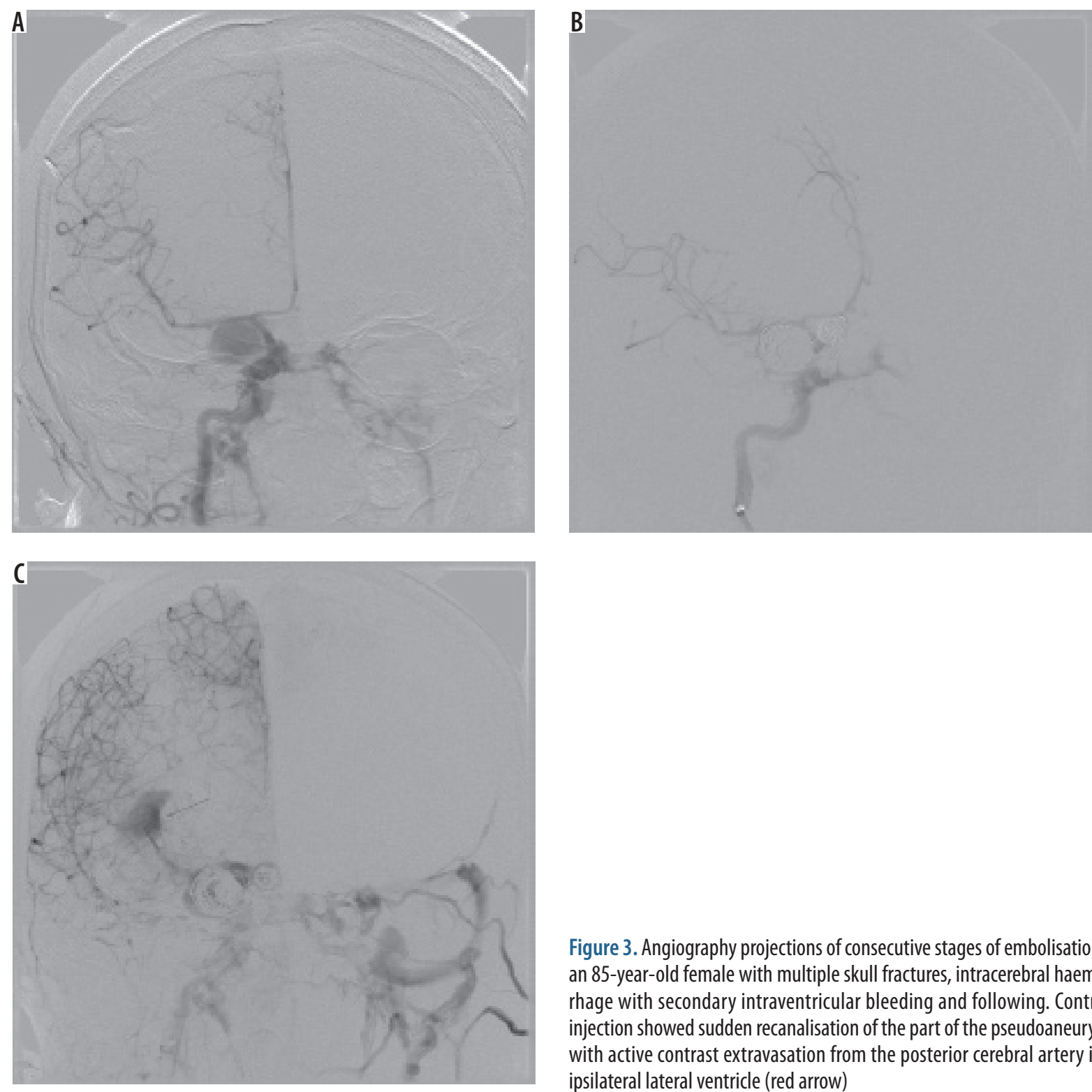

Figure 3. Angiography projections of consecutive stages of embolisation in an 85-year-old female with multiple skull fractures, intracerebral haemorrhage with secondary intraventricular bleeding and following. Contrast injection showed sudden recanalisation of the part of the pseudoaneurysm with active contrast extravasation from the posterior cerebral artery into ipsilateral lateral ventricle (red arrow) 
Alternatively, we used coils, as they have become a mainstay for high flow CCF, in $72 \%$ of cases achieving 92.3\% success rate. A microcatheter could be navigated successfully through a fistulous point, which otherwise would not allow the balloon to pass. Coils are easily controllable, and they can be successfully retrieved and repositioned if the initial placement in not optimal. They are widely available in myriad sizes, rendering collection of necessary hardware easily accessible. Whether the technique involves direct coiling or balloon-/stent-assisted coiling, high blood flow could lead to coil impaction and recanalisation of the fistula $[12,14]$.

Coil embolisation of cavernous sinus poses a technical challenge, which is further increased in cases of posttraumatic pseudoaneurysm rupturing into cavern-
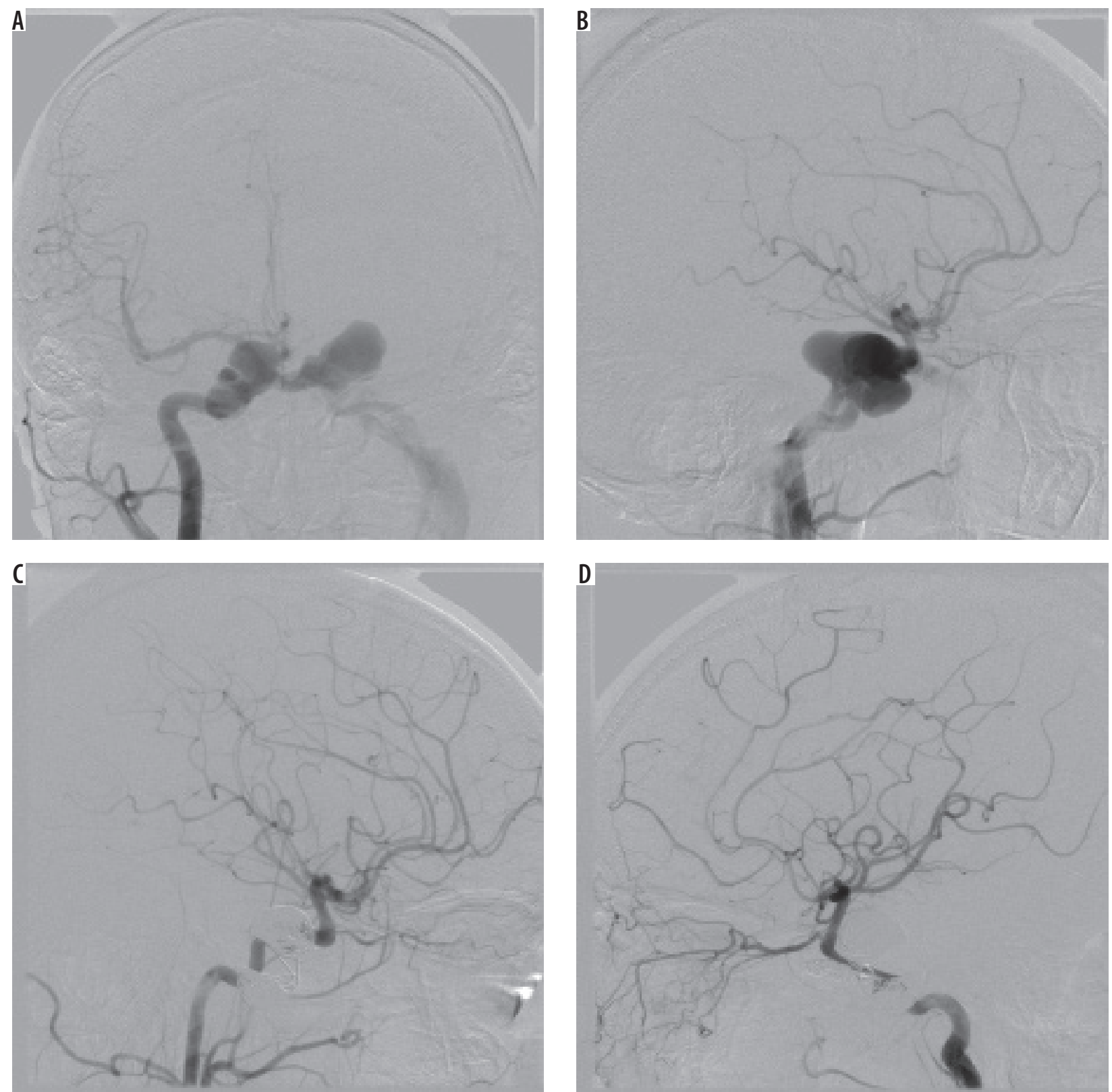

Figure 4. Cerebral angiography of a 24-year-old male with a history of motor vehicle head trauma. Note bilateral giant pseudoaneurysms with concomitant bilateral high-flow carotid-cavernous fistulas (type A). Pseudoaneurysms were gradually embolised with coils, resulting in resolution of fistulas and all symptoms with no peri-procedural complications or neurological deficit 
trast extravasation from the posterior cerebral artery into the ipsilateral lateral ventricle - the patient died eventually of other sustained injuries (Figure 3). The other patient was a 24-year-old male with a history of motor vehicle head trauma, presenting with bilateral exophthalmos, ocular bruit, and chemosis. On cerebral angiography there were bilateral giant pseudoaneurysms with concomitant bilateral high-flow CCFs (type A). Pseudoaneurysms were gradually embolised with coils, resulting in resolution of fistulas and all symptoms with no peri-procedural complications or neurological deficit (Figure 4).

Traumatic intracranial pseudoaneurysms are rare lesions, constituting only $0.15-0.4 \%$ of all intracranial aneurysms, and are usually related to penetrating head trauma or motor vehicle accident [16]. Traumatic CCFs concomitant with ICA pseudoaneurysm are even less frequent [16]. The symptoms could present themselves instantaneously after the injury, as in our first case, or have a latency of many weeks, as the second case exemplifies. Some authors argue that the sac wall of the pseudoaneurysm is not competent enough to withstand the pressure of detachable balloons had they been chosen as a treatment option. On the other hand, gradual placement of soft coils seems to be the treatment of choice for the reasons described above. Care must be taken to avoid any exertion when introducing the coils, as a general rule, which seems even more sensible in the case of post-traumatic pseudoaneurysm [17].

For indirect fistulas, with small and/or multiple fistulous points, we used Onyx-18, an ethylene-vinyl alcohol copolymer mixed with tantalum powder. We achieved a $62.5 \%$ success rate with Onyx-18, which is comparable to other reports $[9,14,15,18,19]$, but inferior to coil embolisation, partly because we assumed those fistulas that we failed to microcatheterise and were bound to be treated with Onyx-18 were marked as failures in our calculations. Other authors used the technique successfully $[15,18,19]$.

Both traumatic and spontaneous CCF could occur bilaterally [20]. In our study group, there were four patients with bilateral CCFs. Three of them had type D bilateral fistulas, and one (the male described above) had a bilateral type A CCF. Most of the bilateral fistulas are indirect in nature; direct CCFs represent the same risk factors as unilateral CCFs [20]. There is a possibility of developing bilateral CCFs of mixed morphology, regarding the classification, i.e. high flow type A combined with moderate/ low flow indirect fistula on contralateral side. Thus, one should follow the general rule of a diagnostic bilateral selective ICA, ECA, and vertebral artery angiography in all patients. A large fistula may mask a smaller contralateral fistula on diagnostic angiography, which may become evident only after occlusion of the former, because a pressure gradient between the ICA and the cavernous sinus is restored. Endovascular treatment of the large or high-flow fistulas should be accompanied by control angiogram of both contralateral ICA and ECA to detect any occult small CCF, especially in patients with bilateral symptomatology and unilateral CCF on initial angiography.

\section{Conclusions}

In this paper, we tried to evaluate successful embolisation of CCFs based on characteristics of SOV and OA. In regard to $\mathrm{SOV}$, successful embolisation was accompanied by resolution of venous reflux, and in cases of highflow fistulas - a decrease in flow velocity. We found that flow normalisation is superior to SOV morphology in post-embolisation assessment. Further investigation is needed with stratification based on the type of fistula. OA was visible in all cases on both pre- and post-embolisation angiographies; thus, its role in angiographic assessment was unremarkable.

Furthermore, we ventured to institute the usefulness of newer endovascular technologies in view of observed departure of established standards. We reported the feasibility of coils and Onyx-18 in treatment of diverse population of patients suffering from carotid-cavernous fistulas, and shared practical insights from our experience.

\section{Ethical approval}

For this type of study formal consent is not required.

\section{Conflict of interest}

The authors report no conflict of interest.

\section{References}

1. Barrow DL, Spector RH, Braun IF, et al. Classification and treatment of spontaneous carotid-cavernous sinus fistulas. J Neurosurg 1985; 62: $248-256$

2. Halbach VV, Hieshima GB, Higashida RT, Reicher M. Carotid cavernous fistulae: indications for urgent treatment. AJR Am J Roentgenol 1987; 149: 587-593.

3. Jacobs SM, Arias EJ, Derdeyn CP, et al. Carotid cavernous sinus fistulas without superior ophthalmic vein enlargement. Ophthalmic Plast Reconstr Surg 2015; 31: 191-196.
4. Ozgen A, Aydingöz U. Normative measurements of orbital structures using MRI. J Comput Assist Tomogr 2000; 24: 493-496.

5. Reis CV, Gonzalez FL, Zabramski JM, et al. Anatomy of the superior ophthalmic vein approach for direct endovascular access to vascular lesions of the orbit and cavernous sinus. Neurosurgery 2009; 64: 318-323; discussion 323.

6. Joshi DK, Singh DD, Garg DD, et al. Assessment of clinical improvement in patients undergoing endovascular coiling in traumatic carotid cavernous fistulas. Clin Neurol Neurosurg 2016; 149: 46-54. 
7. Castillo M, Silverstein M, Hoffman JC, Jr., Barrow D. Spontaneous thrombosis of a direct carotid cavernous sinus fistula: confirmation by Gd-DTPA-enhanced MR. AJNR Am J Neuroradiol 1989; 10: S75-76.

8. Kim MS, Han DH, Kwon OK, et al. Clinical characteristics of dural arteriovenous fistula. J Clin Neurosci 2002; 9: 147-155.

9. Tjoumakaris SI, Jabbour PM, Rosenwasser RH. Neuroendovascular management of carotid cavernous fistulae. Neurosurg Clin N Am 2009; 20: 447-452.

10. Halbach VV, Higashida RT, Hieshima GB, et al. Transvenous embolization of direct carotid cavernous fistulas. AJNR Am J Neuroradiol 1988; 9: 741-747.

11. Kim DJ, Kim DI, Suh SH, et al. Results of transvenous embolization of cavernous dural arteriovenous fistula: a single-center experience with emphasis on complications and management. AJNR Am J Neuroradiol 2006; 27: 2078-2082.

12. Kirsch M, Henkes H, Liebig T, et al. Endovascular management of dural carotid-cavernous sinus fistulas in 141 patients. Neuroradiology 2006; 48: 486-490.

13. Bink A, Goller K, Luchtenberg M, et al. Long-term outcome after coil embolization of cavernous sinus arteriovenous fistulas. AJNR Am J Neuroradiol 2010; 31: 1216-1221.
14. Rodrigues T, Willinsky R, Agid R, et al. Management of dural carotid cavernous fistulas: a single-centre experience. Eur Radiol 2014; 24: 3051-3058.

15. Zhao P, Liu L, Jiang C, et al. Coils and onyx embolization of traumatic carotid-cavernous fistula caused by an intradural internal carotid artery pseudoaneurysm. Neuroradiol J 2012; 25: 231-236.

16. Parkinson D, West M. Traumatic intracranial aneurysms. J Neurosurg 1980; 52: 11-20.

17. Dubey A, Sung WS, Chen YY, et al. Traumatic intracranial aneurysm: a brief review. J Clin Neurosci 2008; 15: 609-612.

18. Ramalingaiah AH, Prasad C, Sabharwal PS, et al. Transarterial treatment of direct carotico-cavernous fistulas with coils and Onyx. Neuroradiology 2013; 55: 1213-1220.

19. Yu Y, Huang Q, Xu Y, et al. Use of onyx for transarterial balloon-assisted embolization of traumatic carotid cavernous fistulas: a report of 23 cases. AJNR Am J Neuroradiol 2012; 33: 1305-1309.

20. Kamel HA, Choudhari KA, Gillespie JS. Bilateral traumatic caroticocavernous fistulae: total resolution following unilateral occlusion. Neuroradiology 2000; 42: 462-465. 